\title{
Quasi-contractions restricted with linear bounded mappings in cone metric spaces
}

Zhilong $\mathrm{Li}^{1,2^{*}}$ and Shujun Jiang ${ }^{3}$

"Correspondence: |z|771218@sina.com

'School of Statistics, Jiangxi University of Finance and

Economics, Nanchang, 330013, China

${ }^{2}$ Research Center of Applied Statistics, Jiangxi University of Finance and Economics, Nanchang, 330013, China

Full list of author information is

available at the end of the article

\begin{abstract}
In this paper, we introduce the notion of a quasi-contraction restricted with a linear bounded mapping in cone metric spaces, and prove a unique fixed point theorem for this quasi-contraction without the normality of the cone. It is worth mentioning that the main result in this paper could not be derived from Ćirić's result by the scalarization method, and hence indeed improves many recent results in cone metric spaces.
\end{abstract}

MSC: $06 \mathrm{~A} 07 ; 47 \mathrm{H} 10$

Keywords: quasi-contraction; cone metric space

\section{Introduction}

Let $(X, d)$ be a complete metric space. Recall that a mapping $T: X \rightarrow X$ is called a quasicontraction, if there exists $\lambda \in(0,1)$ such that

$$
d(T x, T y) \leq \lambda \max \{d(x, y), d(x, T x), d(y, T y), d(x, T y), d(y, T x)\}, \quad \forall x, y \in X .
$$

Ćirić [1] introduced and studied Ćirić's quasi-contractions as one of the most general classes of contractive-type mappings. He proved the well-known theorem that every Ćirić's quasi-contraction $T$ has a unique fixed point. Recently, Ilić and Rakočević [2] generalized this notion to cone metric spaces and extended Ćirićs result to the setting of cone metric spaces, which was then improved by [3, 4]. Afterward, Arandelović and Kečkić [5] considered nonlinear quasi-contractions in cone metric spaces, and proved a fixed point result by the nonlinear scalarization method of $\mathrm{Du}$ [6]. It should be pointed out that Theorem 2.1 of [2], Theorem 2.2 of [3], and Theorem 2.1 of [4] could be derived from Ćirićs result by the nonlinear scalarization method of Du [6]; see (ii) of Remark 2.

In this paper, we introduce the notion of a quasi-contractions restricted with a linear bounded mapping in cone metric spaces. Without using the normality of the cone, we prove the unique existence of fixed point for this quasi-contraction at the expense of

$$
u_{n} \stackrel{w}{\rightarrow} \theta \Rightarrow A u_{n} \stackrel{w}{\rightarrow} \theta, \quad \forall\left\{u_{n}\right\} \subset P .
$$

It is worth mentioning that the main result in this paper could not be derived from Cirić's result by the scalarization method, and hence it indeed improves the corresponding results of [1-4].

○2014 Li and Jiang; licensee Springer. This is an Open Access article distributed under the terms of the Creative Commons Attribution License (http://creativecommons.org/licenses/by/2.0), which permits unrestricted use, distribution, and reproduction in any medium, provided the original work is properly cited. 


\section{Preliminaries}

Let $E$ be a topological vector space. A cone of $E$ is a nonempty closed subset $P$ of $E$ such that $a x+b y \in P$ for each $x, y \in P$ and each $a, b \geq 0$, and $P \cap(-P)=\{\theta\}$, where $\theta$ is the zero element of $E$. A cone $P$ of $E$ determines a partial order $\preceq$ on $E$ by $x \preceq y \Leftrightarrow y-x \in P$ for each $x, y \in X$. In this case $E$ is called an ordered topological vector space.

A cone $P$ of a topological vector space $E$ is solid if int $P \neq \emptyset$, where int $P$ is the interior of $P$. For each $x, y \in E$ with $y-x \in \operatorname{int} P$, we write $x \ll y$. Let $P$ be a solid cone of a topological vector space $E$. A sequence $\left\{u_{n}\right\}$ of $E$ weakly converges [7] to $u \in E$ (denote $u_{n} \stackrel{w}{\rightarrow} u$ ) if for each $\epsilon \in \operatorname{int} P$, there exists a positive integer $n_{0}$ such that $u-\epsilon \ll u_{n} \ll u+\epsilon$ for all $n \geq n_{0}$.

A subset $D$ of a topological vector space $E$ is order-convex if $[x, y] \subset D$ for each $x, y \in D$ with $x \preceq y$, where $[x, y]=\{z \in E: x \preceq z \preceq y\}$. An ordered topological vector space $E$ is order-convex if it has a base of neighborhoods of $\theta$ consisting of order-convex subsets. In this case the cone $P$ is said to be normal. In the case of a normed vector space, this condition means that the unit ball is order-convex, which is equivalent to the condition that there is some positive number $N$ such that $x, y \in E$ and $\theta \preceq x \preceq y$ implies that $\|x\| \leq$ $N\|y\|$, and the minimal $N$ is called a normal constant of $P$. Another equivalent condition is that

$$
\inf \{\|x+y\|: x, y \in P \text { and }\|x\|=\|y\|=1\}>0 .
$$

Then it is not hard to conclude that $P$ is a non-normal cone of a normed vector space $(E,\|\cdot\|)$ if and only if there exist sequences $\left\{u_{n}\right\},\left\{v_{n}\right\} \subset P$ such that

$$
u_{n}+v_{n} \stackrel{\|\cdot\|}{\rightarrow} \theta \quad \nRightarrow \quad u_{n} \stackrel{\|\cdot\|}{\rightarrow} \theta
$$

which implies that the Sandwich theorem does not hold. However, in the sense of weak convergence, the Sandwich theorem still holds even if $P$ is non-normal, and we have the following lemma.

Lemma 1 Let $P$ be a solid cone of a topological vector space $E$ and $\left\{u_{n}\right\},\left\{v_{n}\right\},\left\{z_{n}\right\} \subset E$. If

$$
u_{n} \preceq z_{n} \preceq v_{n}, \quad \forall n,
$$

and there exists some $z \in E$ such that $u_{n} \stackrel{w}{\rightarrow} z$ and $v_{n} \stackrel{w}{\rightarrow} z$, then $z_{n} \stackrel{w}{\rightarrow} z$.

Proof By $u_{n} \stackrel{w}{\rightarrow} w$ and $v_{n} \stackrel{w}{\rightarrow} w$, for each $\epsilon \in \operatorname{int} P$, there exists some positive integer $n_{0}$ such that for all $n \geq n_{0}$,

$$
z-\epsilon \ll u_{n} \quad \text { and } \quad v_{n} \ll z+\epsilon .
$$

Thus we have $z-\epsilon \ll u_{n} \preceq z_{n} \preceq v_{n} \preceq v_{n} \ll z+\epsilon$ for all $n \geq n_{0}$, i.e., $z_{n} \stackrel{w}{\rightarrow} z$. The proof is complete.

The following lemma needed in the further arguments, directly follows from Lemma 1 and Remark 1 of [7].

Lemma 2 Let $P$ be a solid cone of a normed vector space $(E,\|\cdot\|)$. Then for each sequence $\left\{u_{n}\right\} \subset E, u_{n} \stackrel{\|\cdot\|}{\rightarrow} u$ implies $u_{n} \stackrel{w}{\rightarrow} u$. Moreover, if $P$ is normal, then $u_{n} \stackrel{w}{\rightarrow} u$ implies $u_{n} \stackrel{\|\cdot\|}{\rightarrow} u$. 
Let $X$ be a nonempty set and $P$ be a cone of a topological vector space $E$. A cone metric on $X$ is a mapping $d: X \times X \rightarrow P$ such that for each $x, y, z \in X$,

(d1) $d(x, y)=\theta \Leftrightarrow x=y$;

(d2) $d(x, y)=d(y, x)$;

(d3) $d(x, y) \preceq d(x, z)+d(z, y)$.

The pair $(X, d)$ is called a cone metric space over $P$. A cone metric $d$ on $X$ over a solid cone $P$ generates a topology $\tau_{d}$ on $X$ which has a base of the family of open $d$-balls $\left\{B_{d}(x, \epsilon)\right.$ : $x \in X, \theta \ll \epsilon\}$, where $B_{d}(x, \epsilon)=\{y \in X: d(x, y) \ll \epsilon\}$ for each $x \in X$ and each $\epsilon \in \operatorname{int} P$.

Let $(X, d)$ be a cone metric space over a solid cone $P$ of a topological vector space $E$. A sequence $\left\{x_{n}\right\}$ of $X$ converges [7,8] to $x \in X$ (denote by $x_{n} \stackrel{\tau_{d}}{\rightarrow} x$ ) if $d\left(x_{n}, x\right) \stackrel{w}{\rightarrow} \theta$. A sequence $\left\{x_{n}\right\}$ of $X$ is Cauchy [7,8], if $d\left(x_{n}, x_{m}\right) \stackrel{w}{\rightarrow} \theta$. The cone metric space $(X, d)$ is complete [7, 8], if each Cauchy sequence $\left\{x_{n}\right\}$ of $X$ converges to a point $x \in X$.

\section{Main results}

Let $P$ be a solid cone of a normed vector space $(E,\|\cdot\|)$. A mapping $T: X \rightarrow X$ is called a quasi-contraction restricted with a linear bounded mapping, if there exists $u \in$ $\{d(x, y), d(x, T x), d(y, T y), d(x, T y), d(y, T x)\}$ and a linear bounded mapping $A: E \rightarrow E$ with $A(P) \subset P$ such that

$$
d(T x, T y) \leq A u, \quad \forall x, y \in X
$$

Moreover, if $A$ is a contractive mapping (i.e., $A: E \rightarrow E$ is a one-to-one mapping such that $A(P) \subset P, I-A$ is one-to-one and $(I-A)(P) \subset P)$, then $T$ is reduced to the one considered by Arandelović and Kečkić [5]. In particular when $A=\lambda I$ with $\lambda \in(0,1), T$ is an IlićRakočevićs quasi-contraction [2], and also an Arandelović-Kečkić's quasi-contraction.

Remark 1 (i) If $A: E \rightarrow E$ is a mapping such that $A(P) \subset P$ and $(I-A)(P) \subset P$, then it satisfies $(\mathrm{H})$, and so every contractive mapping $A$ satisfies $(\mathrm{H})$. In fact, let $\left\{u_{n}\right\} \subset P$ with $u_{n} \stackrel{w}{\rightarrow} \theta$. By the definition of weak convergence, for each $\epsilon \in \operatorname{int} P$, there exists a positive integer $n_{0}$ such that $\theta \preceq A u_{n} \preceq u_{n} \ll \epsilon$, i.e., $A u_{n} \stackrel{w}{\rightarrow} \theta$.

(ii) If $P$ is normal then every linear bounded mapping $A: P \rightarrow P$ satisfies (H). In fact, let $\left\{u_{n}\right\} \subset P$ with $u_{n} \stackrel{w}{\rightarrow} \theta$. By Lemma 2 and the normality of $P, u_{n} \stackrel{\|\cdot\|}{\rightarrow} \theta$. And so $A u_{n} \stackrel{\|\cdot\|}{\rightarrow} \theta$ for each linear bounded mapping $A: P \rightarrow P$. Moreover, by Lemma $2, A u_{n} \stackrel{w}{\rightarrow} \theta$.

The following example shows that there does exist some linear bounded mapping $A$ : $P \rightarrow P$ satisfying $(\mathrm{H})$ as $P$ is non-normal.

Example 1 Let $E=C_{\mathbb{R}}^{1}[0,1]$ with the norm $\|u\|=\|u\|_{\infty}+\left\|u^{\prime}\right\|_{\infty}$ and $P=\{u \in E: u(t) \geq$ $0, t \in[0,1]\}$ which is a non-normal solid cone [9]. Let $(A u)(t)=\int_{0}^{t} u(s) d s$ for each $u \in P$ and each $t \in[0,1]$.

For each $\varepsilon>0$, there exists some $\epsilon \in \operatorname{int} P$ such that $\|\epsilon\|<\epsilon$. For each $\left\{u_{n}\right\} \subset P$ with $u_{n} \stackrel{w}{\rightarrow}$ $\theta$, there exists a positive integer $n_{0}$ such that for all $n \geq n_{0}, u_{n} \ll \epsilon$, and hence $u_{n}(t) \leq \epsilon(t)$ for each $t \in[0,1]$. Thus we have $\left\|u_{n}\right\|_{\infty} \leq\|\epsilon\|_{\infty} \leq\|\epsilon\|<\varepsilon$ for all $n \geq n_{0}$, i.e., $u_{n} \stackrel{\|\cdot\|_{\infty}}{\rightarrow} \theta$. By the definition of $A$, we get $\left\|A u_{n}\right\|=\left\|A u_{n}\right\|_{\infty}+\left\|\left(A u_{n}\right)^{\prime}\right\|_{\infty}=\left\|A u_{n}\right\|_{\infty}+\left\|u_{n}\right\|_{\infty} \leq 2\left\|u_{n}\right\|_{\infty}$, which together with $u_{n} \stackrel{\|\cdot\|_{\infty}}{\rightarrow} \theta$ implies that $A u_{n} \stackrel{\|\cdot\|}{\rightarrow} \theta$. Thus by Lemma 2, we have $A u_{n} \stackrel{w}{\rightarrow} \theta$. This shows that $A$ satisfies $(\mathrm{H})$. 
In the following, without using the normality of $P$, we show the unique existence of fixed point of quasi-contractions restricted with linear bounded mappings at the expense that $(\mathrm{H})$ is satisfied.

Theorem 1 Let $(X, d)$ be a complete cone metric space over a solid cone $P$ of a normed vector space $(E,\|\cdot\|)$ and $T: X \rightarrow X$ a quasi-contraction restricted with a linear bounded mapping. If the spectral radius $r(A)<1$ and $(\mathrm{H})$ is satisfied, then $T$ has a unique fixed point $x^{*} \in X$ such that for each $x_{0} \in X, x_{n} \stackrel{\tau_{d}}{\rightarrow} x^{*}$, where $x_{n}=T^{n} x_{0}$ for all $n$.

Proof By $r(L)<1$, the inverse of $I-A$ exists, denote it by $(I-A)^{-1}$. Moreover, by Neumann's formula,

$$
(I-A)^{-1}=\sum_{i=0}^{\infty} A^{i}
$$

which together with $A(P) \subset P$ implies that $(I-A)^{-1}(P) \subset P$. It follows from $r(A)<1$ and Gelfand's formula that there exist $0<\beta<1$ and some positive integer $n_{0}$ such that

$$
\left\|A^{n}\right\| \leq \beta^{n}, \quad \forall n \geq n_{0}
$$

We claim that for all $n \geq 1$,

$$
d\left(x_{i}, x_{j}\right) \preceq A(I-A)^{-1} d\left(x_{0}, x_{1}\right), \quad \forall 1 \leq i, j \leq n .
$$

In the following we shall show this claim by induction.

If $n=1$, then $i=j=1$, and so the claim is trivial.

Assume that (4) is true for $n$. To prove that (4) holds for $n+1$, it suffices to show that

$$
d\left(x_{i_{0}}, x_{n+1}\right) \preceq A(I-A)^{-1} d\left(x_{0}, x_{1}\right), \quad \forall 1 \leq i_{0} \leq n .
$$

By (1),

$$
d\left(x_{i_{0}}, x_{n+1}\right) \preceq A u,
$$

where

$$
u \in\left\{d\left(x_{i_{0}-1}, x_{n}\right), d\left(x_{i_{0}-1}, x_{i_{0}}\right), d\left(x_{n}, x_{n+1}\right), d\left(x_{i_{0}-1}, x_{n+1}\right), d\left(x_{n}, x_{i_{0}}\right)\right\} .
$$

Consider the case that $i_{0}=1$.

If $u=d\left(x_{0}, x_{n}\right)$, then by (2), (4), (6), and $A(P) \subset P$,

$$
\begin{aligned}
d\left(x_{i_{0}}, x_{n+1}\right) & \leq A d\left(x_{0}, x_{n}\right) \preceq A\left[d\left(x_{0}, x_{1}\right)+d\left(x_{1}, x_{n}\right)\right] \\
& \preceq A\left[d\left(x_{0}, x_{1}\right)+A(I-A)^{-1} d\left(x_{0}, x_{1}\right)\right] \\
& =A\left(I+A(I-A)^{-1}\right) d\left(x_{0}, x_{1}\right) \\
& =A\left(I+\sum_{i=1}^{\infty} A^{i}\right) d\left(x_{0}, x_{1}\right)=A(I-A)^{-1} d\left(x_{0}, x_{1}\right),
\end{aligned}
$$

i.e., (5) holds. 
If $u=d\left(x_{0}, x_{1}\right)$, then by $(6)$ and $A(P) \subset P$,

$$
d\left(x_{i_{0}}, x_{n+1}\right) \preceq A d\left(x_{0}, x_{1}\right) \preceq \sum_{i=1}^{\infty} A^{i} d\left(x_{0}, x_{1}\right)=A(I-A)^{-1} d\left(x_{0}, x_{1}\right),
$$

i.e., (5) holds.

If $u=d\left(x_{0}, x_{n+1}\right)$, then by $(6)$ and $A(P) \subset P$,

$$
d\left(x_{i_{0}}, x_{n+1}\right) \preceq A d\left(x_{0}, x_{n+1}\right) \preceq A\left[d\left(x_{0}, x_{1}\right)+d\left(x_{i_{0}}, x_{n+1}\right)\right],
$$

which implies that

$$
(I-A) d\left(x_{i_{0}}, x_{n+1}\right) \preceq A d\left(x_{0}, x_{1}\right)
$$

Act on the above inequality with $(I-A)^{-1}$, then by $(I-A)^{-1}(P) \subset P$,

$$
d\left(x_{i_{0}}, x_{n+1}\right) \preceq A(I-A)^{-1} d\left(x_{0}, x_{1}\right),
$$

i.e., (5) holds.

If $u=d\left(x_{n}, x_{i_{0}}\right)$, then by (2), (4), (6), and $A(P) \subset P$,

$$
\begin{aligned}
d\left(x_{i_{0}}, x_{n+1}\right) & \preceq A d\left(x_{i_{0}}, x_{n}\right) \preceq A^{2}(I-A)^{-1} d\left(x_{0}, x_{1}\right) \\
& =\sum_{i=2}^{\infty} A^{i} d\left(x_{0}, x_{1}\right) \preceq \sum_{i=1}^{\infty} A^{i} d\left(x_{0}, x_{1}\right) \\
& =A(I-A)^{-1} d\left(x_{0}, x_{1}\right),
\end{aligned}
$$

i.e., (5) holds.

If $u=d\left(x_{n}, x_{n+1}\right)$, then set $i_{1}=n-1$, and so

$$
d\left(x_{i_{0}}, x_{n+1}\right) \preceq A d\left(x_{i_{1}}, x_{n+1}\right) .
$$

Consider the case that $2 \leq i_{0} \leq n$.

If $u=d\left(x_{i_{0}-1}, x_{n}\right)$, or $u=d\left(x_{i_{0}-1}, x_{i_{0}}\right)$, or $d\left(x_{n}, x_{i_{0}}\right)$, then by (2), (4), (6), and $A(P) \subset P$,

$$
\begin{aligned}
d\left(x_{i_{0}}, x_{n+1}\right) & \preceq A u \preceq A^{2}(I-A)^{-1} d\left(x_{0}, x_{1}\right) \\
& =\sum_{i=2}^{\infty} A^{i} d\left(x_{0}, x_{1}\right) \preceq \sum_{i=1}^{\infty} A^{i} d\left(x_{0}, x_{1}\right) \\
& =A(I-A)^{-1} d\left(x_{0}, x_{1}\right),
\end{aligned}
$$

i.e., (5) holds.

If $u=d\left(x_{n}, x_{n+1}\right)$, or $u=d\left(x_{i_{0}-1}, x_{n+1}\right)$, then set $i_{1}=n$, or $i_{1}=i_{0}-1 \geq 1$, respectively, and so (7) follows.

From the above discussions of both cases, we have the result that either (5) holds, and so the proof of our claim is complete, or there exists $i_{1} \in\{1,2, \ldots, n\}$ such that (7) holds. 
For the latter situation, continue in a similar way, and we will have the result that either

$$
d\left(x_{i_{1}}, x_{n+1}\right) \preceq A(I-A)^{-1} d\left(x_{0}, x_{1}\right),
$$

which together with (7) forces that

$$
d\left(x_{i_{0}}, x_{n+1}\right) \preceq A^{2}(I-A)^{-1} d\left(x_{0}, x_{1}\right) \preceq A(I-A)^{-1} d\left(x_{0}, x_{1}\right),
$$

i.e., (5) holds, and so the proof of our claim is complete, or there exists $i_{2} \in\{1,2, \ldots, n\}$ such that

$$
d\left(x_{i_{1}}, x_{n+1}\right) \preceq A d\left(x_{i_{2}}, x_{n+1}\right) .
$$

If the above procedure ends by the $k$ th step with $k \leq n-1$, that is, there exist $k+1$ integers $i_{0}, i_{1}, \ldots, i_{k} \in\{1,2, \ldots, n\}$ such that

$$
\begin{aligned}
& d\left(x_{i_{0}}, x_{n+1}\right) \preceq A d\left(x_{i_{1}}, x_{n+1}\right), \\
& d\left(x_{i_{1}}, x_{n+1}\right) \preceq A d\left(x_{i_{2}}, x_{n+1}\right), \\
& \ldots, \\
& d\left(x_{i_{k-1}}, x_{n+1}\right) \preceq A d\left(x_{i_{k}}, x_{n+1}\right), \\
& d\left(x_{i_{k}}, x_{n+1}\right) \preceq A(I-A)^{-1} d\left(x_{0}, x_{1}\right),
\end{aligned}
$$

then by (2) and $A(P) \subset P$,

$$
\begin{aligned}
d\left(x_{i_{0}}, x_{n+1}\right) & \leq A^{k+1}(I-A)^{-1} d\left(x_{0}, x_{1}\right)=\sum_{i=k+1}^{\infty} A^{i} d\left(x_{0}, x_{1}\right) \\
& \preceq \sum_{i=1}^{\infty} A^{i} d\left(x_{0}, x_{1}\right)=A(I-A)^{-1} d\left(x_{0}, x_{1}\right),
\end{aligned}
$$

i.e., (5) holds, and so the proof of our claim is complete.

If the above procedure continues more than $n$ steps, then there exist $n+1$ integers $i_{0}, i_{1}, i_{n} \in\{1,2, \ldots, n\}$ such that

$$
\begin{aligned}
& d\left(x_{i_{0}}, x_{n+1}\right) \preceq \operatorname{Ad}\left(x_{i_{1}}, x_{n+1}\right), \\
& d\left(x_{i_{1}}, x_{n+1}\right) \preceq \operatorname{Ad}\left(x_{i_{2}}, x_{n+1}\right), \\
& \ldots, \\
& d\left(x_{i_{n-1}}, x_{n+1}\right) \preceq \operatorname{Ad}\left(x_{i_{n}}, x_{n+1}\right) .
\end{aligned}
$$

It is clear that $i_{0}, i_{1}, i_{n} \in\{1,2, \ldots, n\}$ implies that there exist two integers $k, l \in\{0,1,2, \ldots, n\}$ with $k<l$ such that $i_{k}=i_{l}$, then by (8),

$$
d\left(x_{i_{k}}, x_{n+1}\right) \preceq A^{l-k} d\left(x_{i l}, x_{n+1}\right)=A^{l-k} d\left(x_{i_{k}}, x_{n+1}\right),
$$


and so

$$
\left(I-A^{l-k}\right) d\left(x_{i_{k}}, x_{n+1}\right) \preceq \theta
$$

Note that $r\left(A^{l-k}\right) \leq r(A)^{l-k}<1$, then by Neumann's formula and $A(P) \subset P, I-A^{l-k}$ is invertible (denote its inverse by $\left.\left(I-A^{l-k}\right)^{-1}\right)$, and $\left(I-A^{l-k}\right)^{-1}(P) \subset P$. Acting (9) with $\left(I-A^{l-k}\right)^{-1}$, we get $d\left(x_{i_{k}}, x_{n+1}\right)=\theta$, and hence (5) holds by (9). The proof of our claim is complete.

For all $1<m<n$ and each $x_{0} \in X$, set

$$
C\left(x_{0}, m, n\right)=\left\{d\left(T^{i} x_{0}, T^{j} x_{0}\right): m \leq i, j \leq n\right\} .
$$

From (1), it follows that, for each $u \in C\left(x_{0}, m, n\right)$, there exists some $v \in C\left(x_{0}, m-1, n\right)$ such that $u \preceq A v$. Consequently for all $1<m<n$, there exists $u_{i} \in C\left(x_{0}, m-i, n\right)(i=1,2, \ldots, m-$ 1) such that

$$
d\left(x_{m}, x_{n}\right) \preceq A u_{1} \preceq A^{2} u_{2} \preceq \cdots \preceq A^{m-1} u_{m-1} .
$$

Note that $u_{m-1} \in C\left(x_{0}, 1, n\right)$, which together with (4) implies that

$$
u_{m-1} \preceq A(I-A)^{-1} d\left(x_{0}, x_{1}\right) .
$$

Thus by (10),

$$
d\left(x_{m}, x_{n}\right) \preceq A^{m}(I-A)^{-1} d\left(x_{0}, x_{1}\right), \quad \forall 1<m<n .
$$

It follows from (3) that $\left\|A^{m}\right\| \rightarrow 0(m \rightarrow \infty)$, and hence $A^{m}(I-A)^{-1} d\left(x_{0}, x_{1}\right) \stackrel{\|\cdot\|}{\rightarrow} \theta(m \rightarrow$ $\infty)$, which together with Lemma 2 implies that $A^{m}(I-A)^{-1} d\left(x_{0}, x_{1}\right) \stackrel{w}{\rightarrow} \theta(m \rightarrow \infty)$. Moreover by (11) and Lemma 1, we get

$$
d\left(x_{m}, x_{n}\right) \stackrel{w}{\rightarrow} \theta \quad(n>m \rightarrow \infty)
$$

i.e., $\left\{x_{n}\right\}$ is a Cauchy sequence of $X$. Therefore by the completeness of $X$, there exists some $x^{*} \in X$ such that $x_{n} \stackrel{\tau_{d}}{\rightarrow} x^{*}(n \rightarrow \infty)$, i.e.,

$$
d\left(x_{n}, x^{*}\right) \stackrel{w}{\rightarrow} \theta \quad(n \rightarrow \infty)
$$

By (1),

$$
d\left(T x^{*}, x^{*}\right) \preceq d\left(x_{n+1}, T x^{*}\right)+d\left(x_{n+1}, x^{*}\right) \preceq A u+d\left(x_{n+1}, x^{*}\right), \quad \forall n,
$$

where $u \in\left\{d\left(x_{n}, x^{*}\right), d\left(x_{n}, x_{n+1}\right), d\left(x^{*}, T x^{*}\right), d\left(x_{n}, T x^{*}\right), d\left(x^{*}, x_{n+1}\right)\right\}$.

If $u=d\left(x_{n}, x^{*}\right)$, or $u=d\left(x_{n}, x_{n+1}\right)$, or $u=d\left(x^{*}, x_{n+1}\right)$, then by (12), (13), (14), (H), and Lemma 1, we get $d\left(T x^{*}, x^{*}\right)=\theta$ and hence $x^{*}=T x^{*}$.

If $u=d\left(x^{*}, T x^{*}\right)$, then by (14),

$$
(I-A) d\left(x^{*}, T x^{*}\right) \preceq d\left(x_{n+1}, x^{*}\right), \quad \forall n,
$$


and hence by (13), for each $\epsilon \in \operatorname{int} P$,

$$
(I-A) d\left(x^{*}, T x^{*}\right) \ll \epsilon,
$$

which implies that

$$
(I-A) d\left(x^{*}, T x^{*}\right) \preceq \theta .
$$

Acting (16) with $(I-A)^{-1}$, by $(I-A)^{-1}(P) \subset P$ we get $d\left(T x^{*}, x^{*}\right)=\theta$ and hence $x^{*}=T x^{*}$.

If $u=d\left(x_{n}, T x^{*}\right)$, then by (14), we have

$$
d\left(T x^{*}, x^{*}\right) \preceq d\left(x_{n+1}, x^{*}\right)+A d\left(x_{n}, T x^{*}\right) \preceq d\left(x_{n+1}, x^{*}\right)+A\left[d\left(x_{n}, x^{*}\right)+d\left(x^{*}, T x^{*}\right)\right], \quad \forall n,
$$

and so

$$
(I-A) d\left(x^{*}, T x^{*}\right) \preceq d\left(x_{n+1}, x^{*}\right)+A d\left(x_{n}, x^{*}\right), \quad \forall n .
$$

Thus it follows from (13) and (H) that (15) holds for each $\epsilon \in$ int $P$. Consequently, we obtain (16). Acting (16) with $(I-A)^{-1}$, by $(I-A)^{-1}(P) \subset P$ we get $d\left(T x^{*}, x^{*}\right)=\theta$ and hence $x^{*}=T x^{*}$. This shows that $x^{*}$ is a fixed point of $T$.

If $x$ is another fixed point of $T$, then by (1),

$$
d\left(x, x^{*}\right)=d\left(T x, T x^{*}\right) \preceq A u,
$$

where $u \in\left\{d\left(x, x^{*}\right), d(x, T x), d\left(x^{*}, T x^{*}\right), d\left(x, T x^{*}\right), d\left(x^{*}, T x\right)\right\}$. If $u=d(x, T x)$, or $u=d\left(x^{*}, T x^{*}\right)$, then $u=\theta$, and hence $d\left(x, x^{*}\right)=\theta$. If $u=d\left(x, x^{*}\right)$, or $u=d\left(x, T x^{*}\right)$ or $u=d\left(x^{*}, T x\right)$, then we must have $u=d\left(x, x^{*}\right)$, and hence $(I-A) d\left(x, x^{*}\right) \preceq \theta$. Acting on it with $(I-A)^{-1}$, by $(I-A)^{-1}(P) \subset P$ we have $d\left(x, x^{*}\right)=\theta$. This shows $x^{*}$ is the unique fixed point of $T$. The proof is complete.

Following (i) of Remark 1 and the proof of Theorem 1, we have the following result.

Corollary 1 (see [4, Theorem 2.1]) Let $(X, d)$ be a complete cone metric space over a solid cone $P$ of a topological vector space $E$ and $T: X \rightarrow X$ an Ilić-Rakočević's quasi-contraction. Then $T$ has a unique fixed point $x^{*} \in X$ such that for each $x_{0} \in X, x_{n} \stackrel{\tau_{d}}{\rightarrow} x^{*}$, where $x_{n}=T^{n} x_{0}$ for all $n$.

Remark 2 (i) Theorem 2.1 of [2], Theorem 2.2 of [3], and Theorem 2.1 of [4] are special cases of Theorem 1 with $A=\lambda I$ for $\lambda \in(0,1)$.

(ii) Let $d_{\xi}=\xi_{e} \circ d$, where $\xi_{e}$ is defined by

$$
\xi_{e}(u)=\inf \{r \in \mathbb{R}: u \in r e-P\}, \quad \forall u \in E,
$$

for some $e \in \operatorname{int} P$. Then $d_{\xi}$ is a metric on $X$ by Theorem 2.1 of [6]. If $T$ is a quasicontraction with $\lambda \in(0,1)$, then applying Lemma 1.1 of [6], we have

$$
d_{\xi}(T x, T y) \leq \lambda \max \left\{d_{\xi}(x, y), d_{\xi}(x, T x), d_{\xi}(y, T y), d_{\xi}(x, T y), d_{\xi}(y, T x)\right\}, \quad \forall x, y \in X,
$$


and hence Theorem 2.1 of [2], Theorem 2.2 of [3], and Theorem 2.1 of [4] directly follow from Ćirić's result by Theorem 2.3 of [6].

(iii) If $T$ is a quasi-contraction restricted with a linear bounded mapping, one may not be sure that there exists some $\lambda \in(0,1)$ such that $(17)$ is satisfied, and so Theorem 1 cannot be derived from Ćirić's result. Therefore Theorem 1 indeed improves the corresponding result of [1-4].

(iv) Compared with Corollary 1 of [5], the mapping $A$ is no longer necessarily assumed to be contractive in Theorem 1 .

Remark 3 It is not hard to see from the proof of Theorem 1 that Theorem 1 remains valid in the setting of partial cone metric spaces.

Remark 4 If $A$ is a linear unbounded mapping, then $I-A$ may not be invertible and (3) is not satisfied, and hence the method used in Theorem 1 becomes invalid.

The following example shows the usability of Theorem 1.

Example 2 Let $E$ and $P$ be the same ones as those in Example 1 and $X=P$. Define a mapping $d: X \times X \rightarrow P$ by

$$
\begin{cases}d(x, y)=\theta, & x=y \\ x+y, & x \neq y\end{cases}
$$

Then $(X, d)$ is a complete cone metric space. Let $(T x)(t)=(A x)(t)=\int_{0}^{t} x(s) d s$ for each $x \in X$ and each $t \in[0,1]$. Then from Example 1 we find that $A$ satisfies (H). It is clear that

$$
\begin{cases}d(T x, T y)=\theta=A d(x, y), & x=y, \\ \int_{0}^{t}[x(s)+y(s)] d s=A d(x, y), & x \neq y,\end{cases}
$$

i.e., (1) is satisfied. Since $\left(A^{n} x\right)(t) \leq \frac{t^{n}}{n !}\|x\|_{\infty}$ for each $t \in[0,1]$, then $\left\|A^{n} x\right\|_{\infty} \leq \frac{1}{n !}\|x\|_{\infty}$. Moreover, from $\left(A^{n} x\right)^{\prime}(t)=\left(A^{n-1} x\right)(t)$ we have

$$
\left\|A^{n} x\right\|=\left\|A^{n} x\right\|_{\infty}+\left\|\left(A^{n} x\right)^{\prime}\right\|_{\infty} \leq\left(\frac{1}{n !}+\frac{1}{(n-1) !}\right)\|x\|_{\infty} \leq\left(\frac{1}{n !}+\frac{1}{(n-1) !}\right)\|x\|,
$$

which implies that $\left\|A^{n}\right\| \leq \frac{1}{n !}+\frac{1}{(n-1) !}$. By Gelfand's formula, $r(A)=\lim _{n \rightarrow \infty} \sqrt[n]{\left\|A^{n}\right\|}=0$ since $\lim _{n \rightarrow \infty} \frac{1}{\sqrt[n]{n !}}=0$. Thus all the assumptions of Theorem 1 are satisfied, and hence $T$ has a unique fixed point. In fact, $\theta$ is the unique fixed point of $T$.

Note that if $T$ is not an Ilić-Rakočević's quasi-contraction and $A$ is not a contractive linear bounded mapping (let $x(t)=\cos t$ for each $t \in[0,1]$, then $(A x)(t)=\sin t \geq \cos t=x(t)$ for each $t \in\left[\frac{\pi}{4}, 1\right]$, and so $A x \npreceq x$, which implies that $\left.(I-A)(P) \not \subset P\right)$, then the fixed point results of $[2-5]$ are not applicable in this case. 


\section{Author details}

${ }^{1}$ School of Statistics, Jiangxi University of Finance and Economics, Nanchang, 330013, China. ${ }^{2}$ Research Center of Applied Statistics, Jiangxi University of Finance and Economics, Nanchang, 330013, China. ${ }^{3}$ Department of Mathematics, Jiangxi University of Finance and Economics, Nanchang, 330013, China.

\section{Acknowledgements}

The work was supported by Natural Science Foundation of China (11161022), Natural Science Foundation of Jiangxi Province (20114BAB211006, 20122BAB201015), Educational Department of Jiangxi Province (GJJ12280, GJJ13297) and Program for Excellent Youth Talents of JXUFE (201201). The authors express their gratitude to the referees for their helpful suggestions which improved the proof of Theorem 1.

Received: 26 November 2013 Accepted: 19 March 2014 Published: 03 Apr 2014

\section{References}

1. Cirić, LB: A generalization of Banach's contraction principle. Proc. Am. Math. Soc. 45, 267-273 (1974)

2. Ilić, D, Rakočević, V: Quasi-contraction on a cone metric space. Appl. Math. Lett. 22, 728-731 (2009)

3. Kadelburg, Z, Radenović, S, Rakočević, V: Remarks on 'Quasi-contraction on a cone metric space'. Appl. Math. Lett. 22, 1674-1679 (2009)

4. Rezapour, S, Haghi, RH, Shahzad, N: Some notes on fixed points of quasi-contraction maps. Appl. Math. Lett. 23, 498-502 (2010)

5. Arandelović, ID, Kečkić, DJ: On nonlinear quasi-contractions on TVS-cone metric spaces. Appl. Math. Lett. 24, 1209-1213 (2011)

6. Du, W: A note on cone metric fixed point theory and its equivalence. Nonlinear Anal. 72, 2259-2261 (2010)

7. Jiang, S, Li, Z: Extensions of Banach contraction principle to partial cone metric spaces over a non-normal solid cone. Fixed Point Theory Appl. 2013, Article ID 250 (2013)

8. Huang, LG, Zhang, X: Cone metric spaces and fixed point theorems of contractive mappings. J. Math. Anal. Appl. 332 1468-1476 (2007)

9. Deimling, K: Nonlinear Functional Analysis. Springer, Berlin (1985)

10.1186/1687-1812-2014-87

Cite this article as: Li and Jiang: Quasi-contractions restricted with linear bounded mappings in cone metric spaces. Fixed Point Theory and Applications 2014, 2014:87

\section{Submit your manuscript to a SpringerOpen ${ }^{\circ}$ journal and benefit from:}

- Convenient online submission

- Rigorous peer review

- Immediate publication on acceptance

Open access: articles freely available online

- High visibility within the field

- Retaining the copyright to your article 